\title{
A Favipiravir-induced Fever in a Patient with COVID-19
}

\author{
Takashi Kurita ${ }^{1}$, Keiko Ishida ${ }^{1,2}$, Emiri Muranaka ${ }^{1,2}$, Hiroki Sasazawa ${ }^{1,2}$, Haruki Mito ${ }^{1}$, \\ Yudai Yano ${ }^{1}$ and Ryota Hase ${ }^{1,2}$
}

\begin{abstract}
:
We herein report the first case of a fever induced by favipiravir, a potential coronavirus disease 2019 therapeutic drug. An 82-year-old man diagnosed with bilateral pneumonia was transferred to our hospital following a positive severe acute respiratory syndrome coronavirus 2 polymerase chain reaction test. He was treated with compassionate use of favipiravir. Both his oxygen demand and fever gradually improved after admission; however, his fever relapsed, and the C-reactive protein (CRP) levels increased on day 7. We diagnosed his fever as being favipiravir-induced. The fever resolved a few days after favipiravir discontinuation, demonstrating the accuracy of the diagnosis. This case revealed that favipiravir can induce a fever.
\end{abstract}

Key words: drug fever, favipiravir, coronavirus disease 2019

(Intern Med 59: 2951-2953, 2020)

(DOI: 10.2169/internalmedicine.5394-20)

\section{Introduction}

Coronavirus disease 2019 (COVID-19) has caused a worldwide pandemic. No antiviral therapy has been established for COVID-19, and treatment primarily addresses symptoms. Lopinavir-ritonavir (1) or remdesivir (2) may represent novel antiviral therapies, but their efficacies remain unclear.

Favipiravir was developed as an antiviral agent for influenza (3) and is expected to demonstrate potential efficacy against COVID-19 $(4,5)$. However, data on adverse events are limited, and a drug fever has never been reported.

We herein report the first case of a favipiravir-induced fever during COVID-19 treatment.

\section{Case Report}

An 82-year-old man presented to a primary care clinic with a 3-day fever 6 days prior to admission. He had medical histories of hypertension, colon cancer, and cerebral infarction, and he was on regular medications for levetiracetam, aspirin, amlodipine, and lansoprazole. He was diagnosed with upper respiratory tract infection and given clarithromycin. Because his condition worsened and dyspnea appeared, he visited another hospital 3 days prior to admission. Chest computed tomography (CT) showed bilateral pneumonia; therefore, a SARS-CoV2 polymerase chain reaction (PCR) test was performed. He was treated with ampicillin-sulbactam and azithromycin while awaiting the results. Three days later, he was transferred to our hospital with a positive SARS-CoV2 PCR test.

His vital signs on admission were as follows: blood pressure, $147 / 79 \mathrm{mmHg}$; heart rate, 88 beats/min; body temperature, $37.0{ }^{\circ} \mathrm{C}$; respiratory rate, 30 breaths $/ \mathrm{min}$; and oxygen saturation, $95 \%$, under $12 \mathrm{~L} / \mathrm{min}$ oxygen flow, with a face mask. His physical examination was unremarkable, but his general condition was very poor. Laboratory data showed normal white blood cell counts, reduced lymphocytes, and extremely high lactate dehydrogenase and C-reactive protein levels. Chest $\mathrm{CT}$ showed multiple ground-glass opacities in all lobes of both lungs.

He was started on favipiravir antiviral therapy $(1,600 \mathrm{mg}$ twice the first day and $800 \mathrm{mg}$ twice the second day), under compassionate use, on the day of admission. A few days after admission, his oxygen demand gradually declined, and his low-grade fever improved, suggesting a generally improving condition. However, a fever exceeding $38^{\circ} \mathrm{C}$ appeared 7 days post-admission (Figure). His oxygen demand, general condition, and activities continued to improve, de-

${ }^{1}$ Department of Infectious Diseases, Japanese Red Cross Narita Hospital, Japan and ${ }^{2}$ Department of Infectious Diseases, Kameda Medical Center, Japan

Received for publication May 20, 2020; Accepted for publication August 27, 2020

Correspondence to Dr. Takashi Kurita, takashi_kurita@med.hokudai.ac.jp 


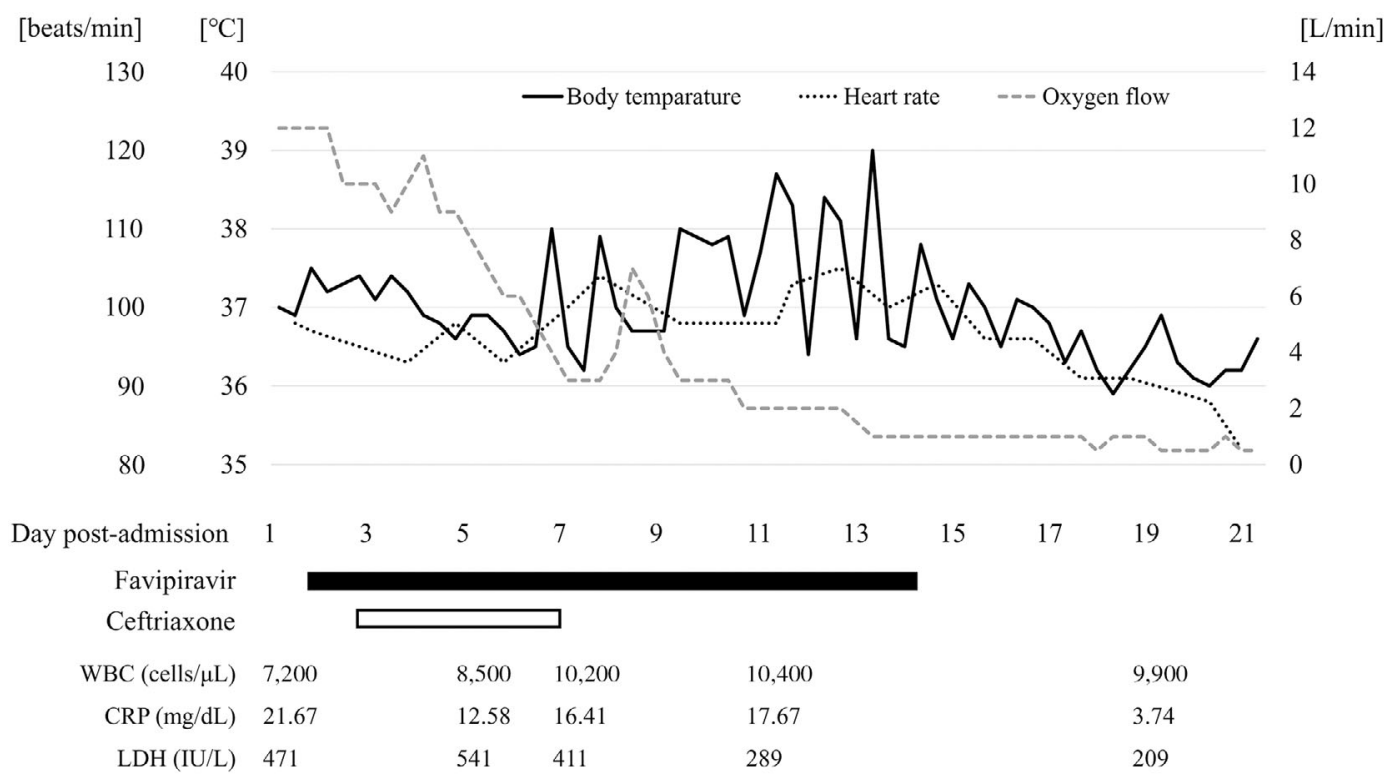

Figure. Clinical course. WBC: white blood cell count, CRP: C-reactive protein, LDH: lactate dehydrogenase

spite the persistent fever (maximum temperature $39.0^{\circ} \mathrm{C}$ ), mild white blood cell count increase, and high $\mathrm{C}$-reactive protein levels. The radiological findings did not improve or worsen. His eosinophil count was 384/ $\mathrm{L}$ (3.7\%), which was within the normal range. No signs of a new infection source were identified in repeated blood and urine cultures. Favipiravir was the only new drug; we therefore diagnosed him with a favipiravir-induced fever and carefully monitored the clinical course.

Favipiravir administration ended on day 14 postadmission, on schedule. The fever improved two days after favipiravir discontinuation and was completely resolved after four days. On day 18 post-admission, his oxygen demand was reduced to nasal $0.5 \mathrm{~L} / \mathrm{min}$. SARS-CoV2 PCR tests performed on day 20 and 21 post-admission were negative. He was discharged, alive, on day 27 post-admission.

According to the adverse drug reaction (ADR) algorithm reported by Naranjo et al. (6), his ADR score was 6, which reflected probable ADR.

Written consent to publish the contents of this case was obtained from the patient in accordance with the Declaration of Helsinki.

\section{Discussion}

Our case suggested that favipiravir can cause a fever as an adverse effect. To our knowledge, a favipiravir-induced fever has not been previously reported, and a fever is not described as an adverse effect in the official manufacturerprovided information.

A drug fever is defined as a fever that develops with the administration of a drug and disappears after discontinuation of the drug when no other cause for the fever can be ascertained after a careful physical examination and appropriate laboratory studies (7). Patients generally take several days to develop a fever following causative drug administration (8), and its resolution typically occurs within 48-72 hours after discontinuation (9).

We concluded that the fever from post-admission day 7 had likely been induced by favipiravir, based on his clinical course (Figure) and an exclusion diagnosis. First, the exacerbation of COVID-19 was unlikely because his fever relapsed over two weeks after the onset of his symptom, and both his oxygen demand and fever gradually improved after admission. The estimated median duration of a fever in patients with COVID-19 is 10 days, and most patients have a normal temperature thereafter (10). His clinical course until postadmission day 6 was consistent with the natural course of COVID-19. Second, no alternative diagnosis was confirmed as a cause of the new-onset fever. Although further examination, such as CT, were not performed, blood and urine cultures remained negative, and no signs of infection were observed by a physical examination. His pulse rate during the high fever that met the definition of relative bradycardia as defined by Cunha et al. (11) may also suggest a druginduced fever rather than a secondary infection. Third, favipiravir is the drug most suspected of having caused a drug fever, given the patient's clinical course. Ceftriaxone was the only drug started after the onset of the disease, except for favipiravir. A fever due to ceftriaxone is rare, occurring in $<1 \%$ (12), and the fever was still present 1 week after drug discontinuation, which is not consistent with the clinical course of a drug fever due to ceftriaxone. His clinical course in which the fever began seven days after favipiravir was started and disappeared four days after its discontinuation strongly suggests that the fever was a favipiravirinduced fever.

Furthermore, the ADR score proposed by Naranjo et al. was suggestive of drug-related side effects in this case. The ADR score is widely used as a criterion for assessing the 
validity of a drug's side effects, specifically to determine whether they are actually caused by the drug or by other factors. In our case, a score of 6 was calculated, which was considered indicative of "probable ADR" because the adverse event occurred after favipiravir was administered (+2), the symptoms improved after the drug was discontinued $(+1)$, no other cause was found $(+2)$, and the adverse event was an objective finding of temperature $(+1)$.

Our report can help assess the clinical course of patients with COVID-19 treated with favipiravir. A previous study reported that a fever may appear after admission in patients with COVID-19 (13). In addition, secondary infection occurs often in patients with COVID-19 (14). These findings suggest that the assessment of a new-onset fever during treatment is important for judging the clinical improvement in COVID-19. Keeping in mind the possibility of a favipiravir-induced fever may help avoid the unnecessary administration of antiviral or antimicrobial treatment. More cases of a favipiravir-induced fever may appear once the efficacy of favipiravir against COVID-19 is established.

In conclusion, favipiravir may cause a fever as an adverse effect, and a drug fever should be considered when a fever appears during favipiravir treatment in patients with COVID-19.

The authors state that they have no Conflict of Interest (COI).

\section{References}

1. Cao B, Wang Y, Wen D, et al. A trial of lopinavir-ritonavir in adults hospitalized with severe Covid-19. N Engl J Med 382: 1787-1799, 2020

2. Grein J, Ohmagari N, Shin D, et al. Compassionate use of rem- desivir for patients with severe Covid-19. N Engl J Med 382: 2327-2336, 2020

3. Furuta $\mathrm{Y}$, Takahashi $\mathrm{K}$, Fukuda $\mathrm{Y}$, et al. In vitro and in vivo activities of anti-influenza virus compound T-705. Antimicrob Agents Chemother 46: 977-981, 2002.

4. Lu CC, Chen MY, Chang YL. Potential therapeutic agents against COVID-19: what we know so far. J Chin Med Assoc 83: 534-536, 2020.

5. Tu YF, Chien CS, Yarmishyn AA, et al. A review of SARS-CoV-2 and the ongoing clinical trials. Int J Mol Sci 21: 2657, 2020.

6. Naranjo CA, Busto U, Sellers EM, et al. A method for estimating the probability of adverse drug reactions. Clin Pharmacol Ther 30: 239-245, 1981.

7. Mackowiak PA, LeMaistre CF. Drug fever: a critical appraisal of conventional concepts. An analysis of 51 episodes in two Dallas hospitals and 97 episodes reported in the English literature. Ann Intern Med 106: 728-733, 1987.

8. Lipsky BA, Hirschmann JV. Drug fever. JAMA 245: 851-854, 1981.

9. Patel RA, Gallagher JC. Drug fever. Pharmacotherapy 30: 57-69, 2010.

10. Chen J, Qi T, Liu L, et al. Clinical progression of patients with COVID-19 in Shanghai, China. J Infect 80: e1-e6, 2020.

11. Cunha BA. Diagnostic significance of relative bradycardia. Infect Dis Pract 21: 38-40, 1997.

12. Grayson ML, Cosgrove SE, Mccarthy JS, et al. Kucers' The Use of Antibiotics: A Clinical Review of Antibacterial, Antifungal, Antiparasitic, and Antiviral Drugs. 7th ed. CRC Press, Boca Raton, FL, 2017: 483 .

13. Guan W, Ni Z, Hu Y, et al. Clinical characteristics of coronavirus disease 2019 in China. N Engl J Med 382: 1708-1720, 2020.

14. Zhou F, Yu T, Du R. Clinical course and risk factors for mortality of adult inpatients with COVID-19 in Wuhan, China: a retrospective cohort study. Lancet 395: 1054-1062, 2020.

The Internal Medicine is an Open Access journal distributed under the Creative Commons Attribution-NonCommercial-NoDerivatives 4.0 International License. To view the details of this license, please visit (https://creativecommons.org/licenses/ by-nc-nd/4.0/).

(C) 2020 The Japanese Society of Internal Medicine Intern Med 59: 2951-2953, 2020 